\title{
Evodiamine suppresses capsaicin-induced thermal hyperalgesia through activation and subsequent desensitization of the transient receptor potential $\mathrm{V} 1$ channels
}

\author{
Emiko Iwaoka $^{1}$ Shenglan Wang ${ }^{1,2} \cdot$ Nobuyuki Matsuyoshi $^{1,4} \cdot$ Yoko Kogure $^{1} \cdot$ \\ Shunji Aoki ${ }^{1} \cdot$ Satoshi Yamamoto $^{1} \cdot$ Koichi Noguchi $^{2,3} \cdot$ Yi Dai $^{1,2,3}$
}

Received: 21 April 2015/Accepted: 5 July 2015/Published online: 19 July 2015

(C) The Japanese Society of Pharmacognosy and Springer Japan 2015

\begin{abstract}
Evodiae fructus (EF), a fruit of Evodia rutaecarpa Bentham, has long been used as an analgesic drug in traditional Chinese and Japanese medicine. However, the underlying molecular mechanism of its pharmacological action is unclear. Here, using calcium imaging, whole-cell patch-clamp recording, and behavioral analysis, we investigated the pharmacological action of EF and its principal compound, evodiamine, on the transient receptor potential (TRP) V1 channels. Dorsal root ganglion (DRG) neurons and TRPV1- or TRPA1-transfected human embryonic kidney-derived (HEK) 293 cells were used for calcium imaging or whole-cell patch-clamp recording. Twenty male adult Sprague-Dawley rats were used for the capsaicininduced thermal hyperalgesia behavioral analyses. We found that evodiamine induced significant increases in intracellular calcium and robust inward currents in a subpopulation of isolated rat DRG neurons, most of which were also sensitive to capsaicin. The effect of evodiamine was completely blocked by capsazepine, a competitive antagonist of TRPV1. Evodiamine induced significant inward currents in TRPV1-, but not TRPA1-transfected
\end{abstract}

E. Iwaoka and S. Wang contributed equally.

Yi Dai

ydai@huhs.ac.jp

1 Department of Pharmacy, School of Pharmacy, Hyogo University of Health Sciences, 1-3-6 Minatojima, Chuo-ku, Kobe, Hyogo 6508530, Japan

2 Traditional Medicine Research Center, Chinese Medicine Confucius Institute, Hyogo College of Medicine, Kobe, Hyogo 6508530, Japan

3 Department of Anatomy and Neuroscience, Hyogo College of Medicine, Nishinomiya, Hyogo 6638501, Japan

4 Present Address: FALCO Pharmacies Ltd., Kyoto, Japan
HEK293 cells. Pretreatment with evodiamine reduced capsaicin-induced currents significantly. Furthermore, the in vivo pre-treatment of evodiamine suppressed thermal hyperalgesia induced by intraplantar injection of capsaicin in rats. These results identify that the analgesic effect of EF and evodiamine may be due to the activation and subsequent desensitization of TRPV1 in sensory neurons.

Keywords Wu-Zhu-Yu - Goshuyu · Evodiamine · TRPV1 · Desensitization · Hyperalgesia

\section{Introduction}

The transient receptor potential channel subfamily $\mathrm{V}$ member 1 (TRPV1), also known as the capsaicin receptor, is expressed by a subset of the small-sized dorsal root ganglion (DRG) or trigeminal ganglia neurons [1]. TRPV1 is activated by capsaicin, protons, or noxious heat (with a thermal threshold $>43{ }^{\circ} \mathrm{C}$ ), which causes pain in vivo [1, 2]. TRPV1 selective antagonists can inhibit mechanotransmission in primary sensory neurons following inflammation [3]. In addition, TRPV1 can be sensitized by proinflammatory agents such as prostaglandins, bradykinin, adenosine triphosphate (ATP), protease activated receptors (PAR) 2, nerve growth factor (NGF), and tumor necrosis factor alpha (TNF- $\alpha$ ), which directly or indirectly modulate the channel protein or the probability of channel opening by stimuli [4-8]. Analyses of mice lacking TRPV1 have shown that it is essential for selective modalities of pain sensation as well as tissue injury-induced thermal hyperalgesia [9]. Thus, TRPV1 functions as a molecular integrator of painful stimuli in which each stimulus sensitizes the channel to other stimuli, with the end result that TRPV1 
acts as a molecular amplifier in the sensory neuron. These insights have renewed the interest in TRPV1 as an important site of analgesia.

Evodiae fructus (EF) is one of the most popular and multi-purpose herbs traditionally used in China (known as Wu-Zhu-Yu) and Japan (Goshuyu) for the treatment of headaches, abdominal pain, difficult menstruation, vomiting, diarrhea, and other diseases [10]. Evodiamine (Fig. 1), a natural indole alkaloid, is the major bioactive constituent of EF. Evodiamine has been reported to possess multiple biological effects such as antinociceptive, antiinflammatory, antineoplastic, antidiabetic, and thermoregulatory [11-15]. However, the molecular mechanism underlying evodiamine's effect on antinociception remains unclear. Pearce and coworkers have characterized evodiamine as an agonist for rat TRPV1 expressed heterologously in $\mathrm{CHO}$ cells using calcium uptake analysis [16]. However, whether evodiamine directly excites TRPV1 in sensory neurons has not been clarified yet. Since activation of TRPV1 may produce a nociceptive response, it is of interest whether and how evodiamine exerts its antinociceptive effect through TRPV1 activation, and this is important for explaining the effect of EF in pain-related diseases.

To the best of our knowledge, we report for the first time that evodiamine activates TRPV1 in sensory neurons. Our patch-clamp analyses indicate that the antinociceptive effect of evodiamine may be attributed to the evodiamineinduced activation and subsequent desensitization of TRPV1.

\section{Materials and methods}

\section{Mammalian cell culture}

HEK 293 cells were maintained in Dulbecco's Modified Eagle's Medium (DMEM), supplemented with $10 \%$ fetal bovine serum (FBS), $2 \mathrm{mM}$ glutamax, penicillin, and streptomycin. HEK293 cells were transfected with $1 \mu \mathrm{g}$ human TRPA1 (hTRPA1) cDNA or rat TRPV1 (rTRPV1) cDNA using Lipofectamine LTX and PLUS Reagent (Invitrogen, Carlsbad, CA, USA). An enhanced green

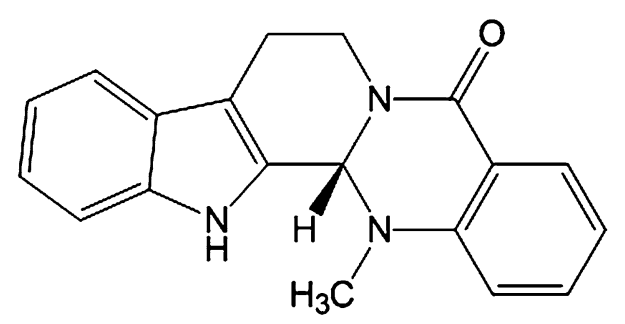

Fig. 1 Chemical structure of evodiamine fluorescence protein reporter plasmid (BD Biosciences, San Jose, CA, USA) was cotransfected with the TRP channels. The hTRPA1 and rTRPV1 cDNAs were generous gifts from Prof. Makoto Tominaga (Okazaki Institute of Integrative Bioscience, Okazaki, Japan). For primary cultures of DRG neurons, DRGs were collected from adult Sprague-Dawley rats (100-150 g) using sterile techniques and placed in ice-cold Earle's Balanced Salt Solution (EBSS, Sigma-Aldrich Co. LLC, St Louis, MO, USA). The adhering fat and connective tissue were removed, and each DRG preparation was immediately placed in a medium consisting of $2 \mathrm{ml}$ EBSS and $1.25 \mathrm{mg} / \mathrm{ml}$ collagenase $\mathrm{P}$ (Sigma-Aldrich Co. LLC, St Louis, MO, USA) and kept at $37{ }^{\circ} \mathrm{C}$ for $60 \mathrm{~min}$ with occasional agitation. After dissociation of the DRG cells, the cell suspension was centrifuged for $5 \mathrm{~min}$ at $250 \times g$, and the cell pellet was resuspended in EBSS supplemented with $10 \% \mathrm{FBS}, 2 \mathrm{mM}$ glutamax and glucose, $1 \times$ MEM vitamin, penicillin, and streptomycin. Recombinant rat NGF (100 ng/ml, Sigma-Aldrich Co. LLC, St Louis, MO, USA) was added to the medium.

\section{Calcium imaging}

Ratiometric calcium imaging was performed using an Olympus fluorescence microscope (IX 70, Olympus) equipped with an Orca-ER digital CCD camera (Hamamatsu Photonics, Shizuoka, Japan). Dual images (340 and $380 \mathrm{~nm}$ excitation, $510 \mathrm{~nm}$ emission) were collected, and pseudocolor ratiometric images were monitored every $5 \mathrm{~s}$ during the experiment using a ratio imaging system (AQUACOSMOS/Ratio, Hamamatsu Photonics, Shizuoka, Japan). Rat DRG neurons were cultured on poly-L-lysinecoated glass coverslips for 18-24 h and were subsequently loaded with $2 \mu \mathrm{M}$ Fura-2 acetoxymethyl ester (Fura-2 AM) for $40 \mathrm{~min}$ at $37^{\circ} \mathrm{C}$. DRG neurons in one randomly selected microscopic field (10-30 cells) on one glass coverslip were measured.

\section{Electrophysiology}

Whole-cell patch-clamp recordings were performed 2 days after transfection of HEK293 cells with hTRPA1 cDNA or 1 day after transfection with rTRPV1 cDNA or DRG neuron culture. Voltage-clamp experiments were performed at a $-60 \mathrm{mV}$ holding potential, and recordings were sampled at $5 \mathrm{kHz}$ and filtered at $2 \mathrm{kHz}$. In all tests, agonists were applied until the evoked currents underwent desensitization. In all experiments, the current magnitude was quantified by the peak current amplitude. The standard bath solution contained $140 \mathrm{mM} \mathrm{NaCl}, 5 \mathrm{mM} \mathrm{KCl}$, $2 \mathrm{mM} \mathrm{MgCl}_{2}, 2 \mathrm{mM} \mathrm{CaCl}_{2}, 10 \mathrm{mM}$ HEPES, and $10 \mathrm{mM}$ glucose, $\mathrm{pH} 7.4$ (adjusted with $\mathrm{NaOH}$ ). The pipette solution contained $140 \mathrm{mM} \mathrm{KCl}, 2 \mathrm{mM} \mathrm{MgCl}_{2}, 0.5 \mathrm{mM} \mathrm{CaCl}$, 
5 mM Mg-ATP, 5 mM EGTA, and 10 mM HEPES, pH 7.2 (adjusted with $\mathrm{KOH}$ ). All patch-clamp experiments were performed at room temperature (RT, $\sim 25^{\circ} \mathrm{C}$ ). The solutions containing drugs were applied to the chamber $(1 \mathrm{ml})$ by a gravity system at a flow rate of $3-4 \mathrm{ml} / \mathrm{min}$.

\section{Behavior studies}

Twenty male adult Sprague-Dawley rats (200-250 g) were used for the behavioral analyses. After adaptation, each rat received an intraplantar injection of $50 \mu \mathrm{l}$ evodiamine $(100 \mu \mathrm{M}$ in $5 \%$ DMSO and $0.5 \%$ Tween- 20 in saline) or vehicle (5\% DMSO and $0.5 \%$ Tween-20 in saline) into the left hind paw. At $10 \mathrm{~min}$ after injection, rats received an intradermal injection of $50 \mu \mathrm{l}$ capsaicin $(220 \mu \mathrm{M}, 0.5 \%$ Tween-20 in saline) or vehicle $(0.5 \%$ Tween-20 in saline) in the same area as the evodiamine injection. For the thermal hyperalgesia analysis, the response latencies to a radiant paw heating were measured after injection of capsaicin at $0,10,30,60$, and $120 \mathrm{~min}$ using the plantar test (Ugo Basile, Comerio, Italy) as described previously [6]. Briefly, a radiant heat source beneath a glass pane was aimed at the planter surface of the hindpaw. Two latency measurements were taken for each ipsilateral hindpaw in each test session. The hindpaws were tested alternately, with 5-min intervals between consecutive tests. The two latency measurements per side were averaged. The heat stimulus was terminated after a withdrawal response or after $30 \mathrm{~s}$ to avoid skin damage. The ratio of the recording at $10,30,60$, or $120 \mathrm{~min}$ after injection to that before the injection in each rat was used for statistical analysis. A researcher who was unaware of the treatment group performed all the behavioral experiments. All procedures involving the care and use of animals were approved by the Hyogo University of Health Sciences Committee on Animal Research and were carried out in accordance with the NIH guidelines for the care and use of laboratory animals.

\section{Plant material and extract preparation}

EF (produced in Jiangxi Province, China, lot no. 003807001), the fruit of Evodia rutaecarpa Bentham, E. officinalis Dode, or E. bodinieri Dode, was purchased from Tochimoto Tenkaido Co., Ltd. (Osaka, Japan) and kept in the laboratory of Hyogo University of Health Sciences at room temperature. The EF was extracted with $\mathrm{MeOH}$ and evaporated in vacuo to obtain $\mathrm{MeOH}$ extract.

\section{Compounds}

Allyl isothiocyanate (AITC), Fura-2 AM, and capsaicin were from Nacalai Tesque (Kyoto, Japan). Evodiamine was from Alexis Corp. (Lausen, Switzerland). Capsazepine was from Sigma-Aldrich Co., LLC (St Louis, MO, USA). Glutamax, FBS, penicillin-streptomycin, MEM vitamin solution, and OPTI-MEM were from Invitrogen (Carlsbad, CA, USA).

\section{Statistical analysis}

All results are expressed as mean \pm SEM. An unpaired $t$ test was used to compare the electrophysiological data between the two groups. Two-way repeated measures ANOVA followed by Fisher's PLSD was applied to the behavioral data. A difference was accepted as significant if the probability was $<5 \%(p<0.05)$.

\section{Results and discussion}

\section{Evodiamine activated TRPV1 in sensory neurons}

Using calcium imaging, we found that either the extract of EF or evodiamine caused a rapid elevation in intracellular calcium levels in a subpopulation of small-sized isolated rat DRG neurons; almost all of the evodiamine-sensitive neurons also responded to capsaicin (Fig. 2a-c). In the whole-cell patch clamp recording, evodiamine induced robust inward currents in the capsaicin-sensitive DRG neurons (Fig. 2d). The evodiamine-induced intracellular calcium response was completely blocked by capsazepine (Fig. 3a, b). These data suggested that the traditional herbal medicine EF might directly excite primary sensory neurons through the evodiamine-induced activation of TRPV1, providing the first evidence for evodiamine-induced sensory neuron activation. A previous study has reported that evodiamine induced calcium uptake in TRPV1-expressing CHO cells, which showed the same interaction of evodiamine and TRPV1 but in heterologous cells [16]. In the present study, capsazepine completely blocked the evodiamine-induced calcium influx, suggesting that evodiamine may bind to TRPV1 at the same binding site with capsaicin. This idea is also supported by other studies [16, 17].

\section{Evodiamine activated and subsequently desensitized TRPV1 in heterologous HEK cells}

To further determine the agonistic action of evodiamine on TRPV1, we examined the evodiamine-activated currents in TRPV1- or TRPA1-transfected HEK293 cells. Evodiamine $(10 \mu \mathrm{M})$ did not evoke any current in untransfected or TRPA1-transfected HEK293 cells as measured by voltage clamp recordings (data not shown and Fig. 4a, b).

Pharmacological desensitization of receptors is a fundamental approach for reducing neuronal activity. We thus 
a
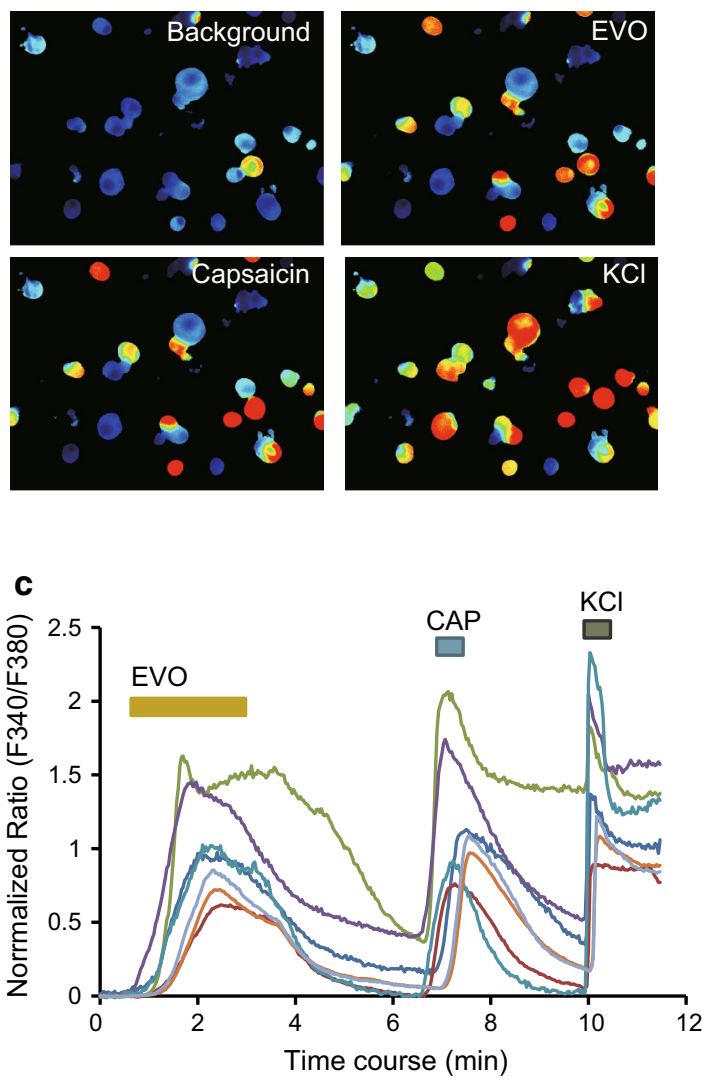

Fig. 2 EF- and evodiamine-induced activation in DRG neurons. a Representative fluorescence images of the evodiamine-induced changes in the intracellular calcium concentration. The pseudocolor indicates the fluorescence intensity at an excitation and emission of $340 / 380$ and $510 \mathrm{~nm}$, respectively. b Time courses of representative traces from individual cells show EF extract $(100 \mu \mathrm{g} / \mathrm{ml})$ and capsaicin $(1 \mu \mathrm{M})$-induced changes in the intracellular calcium concentration. $\mathbf{c}$ Time courses of representative traces from individual

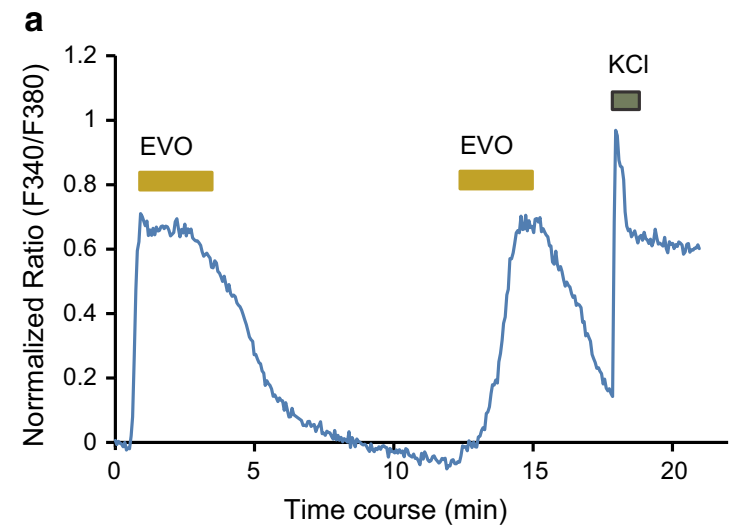

Fig. 3 Evodiamine-induced calcium influx through TRPV1 in DRG neurons. a, $\mathbf{b}$ The time courses of the representative traces show the changes in the intracellular calcium concentration induced by repeated application of evodiamine $(3 \mu \mathrm{M})$. The second application of evodiamine is performed in the absence (a) and presence (b) of capsazepine $(10 \mu \mathrm{M})$, a selective TRPV1 antagonist, respectively. b

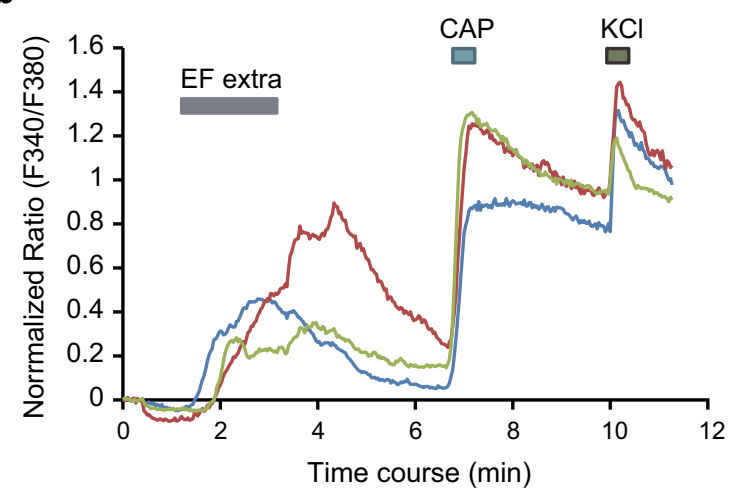

d

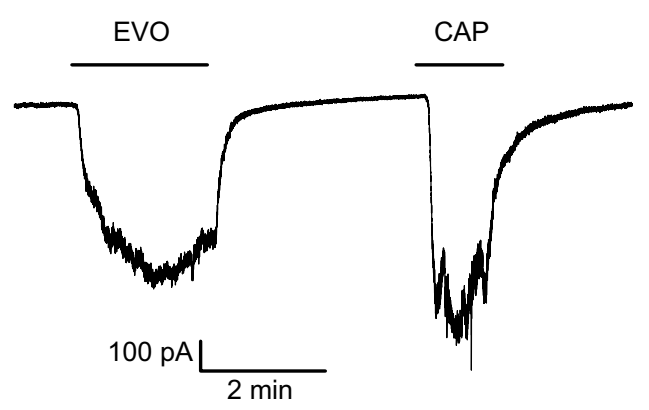

cells show evodiamine $(3 \mu \mathrm{M})$ and capsaicin $(1 \mu \mathrm{M})$-induced changes in the intracellular calcium concentration. d Representative trace shows the evodiamine $(10 \mu \mathrm{M})$ and capsaicin $(1 \mu \mathrm{M})$-induced inward current in DRG neuron. The horizontal bars indicate the duration of the compound application. The holding potential is $-60 \mathrm{mV}$ in patchclamp recording. All of the neuron that responds to $\mathrm{KCl}(50 \mathrm{mM})$ is used for analysis in calcium imaging. EF extra EF extract, EVO evodiamine, $C A P$ capsaicin

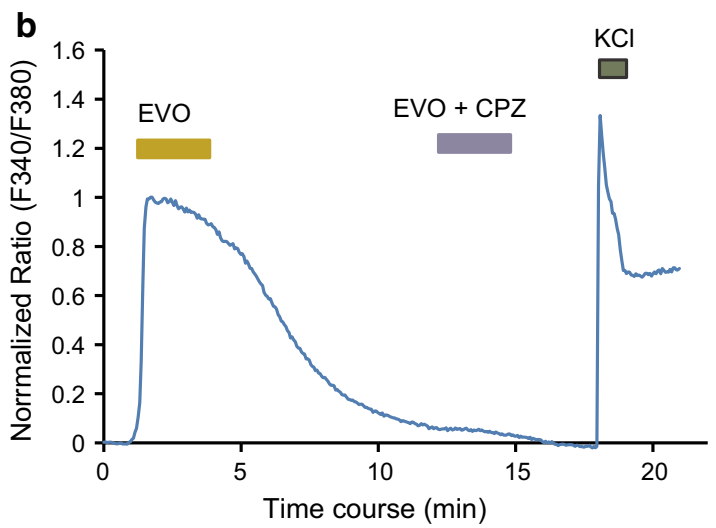

The horizontal bars indicate the duration of the compound application. Note that capsazepine completely blocks the EVO-induced calcium influx. All of the neuron that responds to $\mathrm{KCl}(50 \mathrm{mM})$ is used for analysis in calcium imaging. $E V O$ evodiamine, $C P Z$ capsazepine 
a

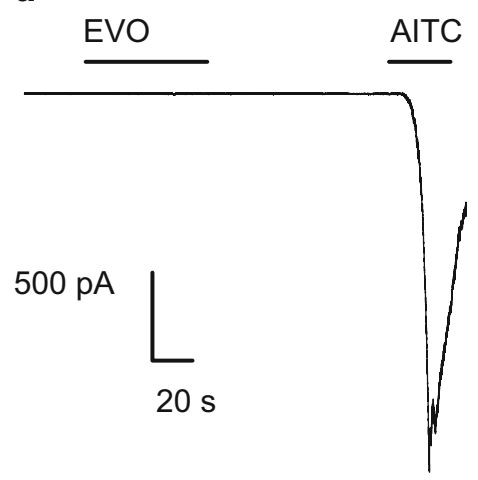

Fig. 4 Evodiamine activates TRPV1 but not TRPA1 in heterologous HEK293 cells. Representative traces from whole-cell patch-clamp experiments show the evodiamine $(10 \mu \mathrm{M})$-induced currents in an hTRPA1- (a) and rTRPV1-transfected HEK293 cell (b). In each experiment, a well-known agonist of TRPA1 or TRPV1 was applied

examined the effects of pretreatment with evodiamine on the capsaicin-induced currents in TRPV1-expressing HEK293 cells. We found that a single application of $100 \mathrm{nM}$ capsaicin (without evodiamine pretreatment) induced robust inward currents with a current density of $-327.4 \pm 61.3 \mathrm{pA} / \mathrm{pF}(n=12)$, which were significantly inhibited by pretreatment with $1 \mu \mathrm{M}$ evodiamine $(-132.5 \pm 16.2 \mathrm{pA} / \mathrm{pF} ; n=6 ; p<0.05$ vs. capsaicin) (Fig. 5a, b). These observations indicated that pretreatment with evodiamine could desensitize the channel activity of TRPV1 to capsaicin. Evodiamine has been suggested to sensitize and desensitize the capsaicin-sensitive sensory afferents in mice [18]. Here, we first demonstrated at the

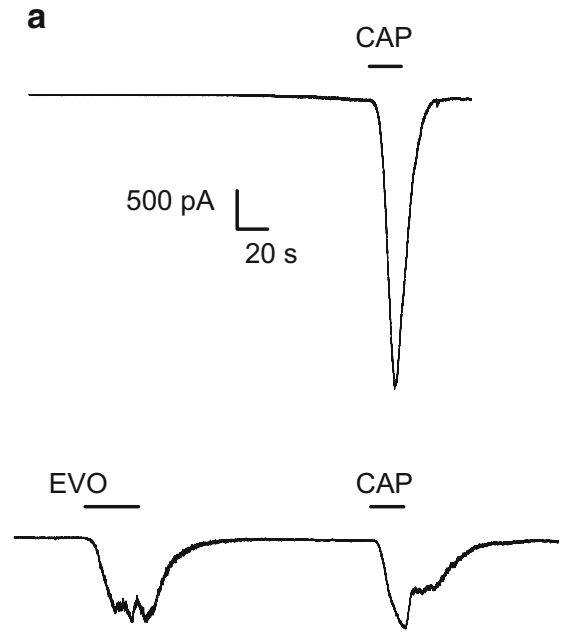

Fig. 5 Evodiamine suppressed capsaicin-induced currents by desensitizing TRPV1 in heterologous HEK293 cells. a Representative traces from the whole-cell recordings show the capsaicin $(100 \mathrm{nM})$ induced currents in the absence (upper) or presence (lower) of a pretreatment with $1 \mu \mathrm{M}$ evodiamine. The horizontal bars indicate the duration of the compound application. b Bar graph shows the effect b

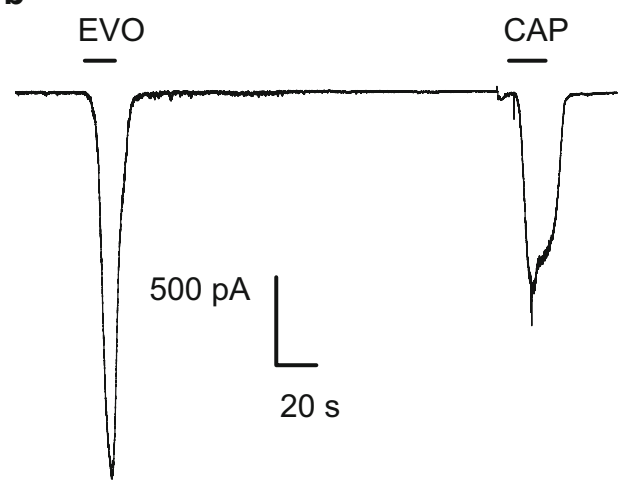

at the end of the recording. Note that evodiamine did not induce any currents in TRPA1-transfected cells. The horizontal bars indicate the duration of the compound application. The holding potential was $-60 \mathrm{mV}$ in all experiments. EVO evodiamine, AITC allyl isothiocyanate, $C A P$ capsaicin

cellular level that evodiamine could activate and then subsequently desensitize the TRPV1 channel.

\section{Evodiamine reduced capsaicin-induced thermal hyperalgesia}

Activation of TRPV1 by pungent natural products suggests a nociceptive role for TRPV1. Desensitization of TRPV1 channels shows a therapeutic value in pain relief [19]. Several TRPV1 agonists have been tried for pain therapy in the clinical practice [20, 21]. Due to its agonism, evodiamine activated and then desensitized TRPV1 in our present in vitro experiments. To further validate whether

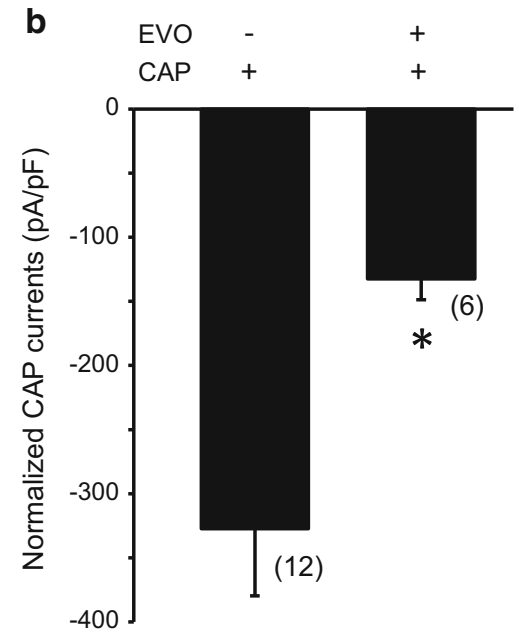

of pretreatment of evodiamine $(1 \mu \mathrm{M})$ on capsaicin $(100 \mathrm{nM})$ induced TRPV1 currents. Data are generated from separate cells in whole cell recordings. $N=6-10$ cells in each time experiment. The holding potential is $-60 \mathrm{mV}$ in all experiments. $C A P$ capsaicin, $E V O$ evodiamine 
evodiamine application in vivo can also suppress the TRPV1-mediated pain behavior, we administered intraplantar injections of evodiamine followed by capsaicin injections to Sprague-Dawley rats and recorded their behavior toward capsaicin-induced thermal hyperalgesia. Consistent with our previous studies [6, 22], the capsaicin injection induced a significant and rapid thermal hyperalgesia at the site of injection that vanished $2 \mathrm{~h}$ post injection. Interestingly, the injection of evodiamine $(50 \mu \mathrm{l}$, $100 \mu \mathrm{M}$ in $5 \%$ DMSO, and $0.5 \%$ Tween-20 in saline) itself did not cause acute nocifensive behavior such as paw lifting, flinching, or licking. This lack of an irritant effect of evodiamine may be due to its low potency and efficacy for activating TRPV1. At $10 \mathrm{~min}$ after evodiamine or vehicle treatment, capsaicin was injected into the same area of the hind paw. As expected, the pretreatment with evodiamine induced a significant decrease in the withdrawal latency of the hind paw $30 \mathrm{~min}$ after the intraplantar capsaicin injection (Fig. 6).

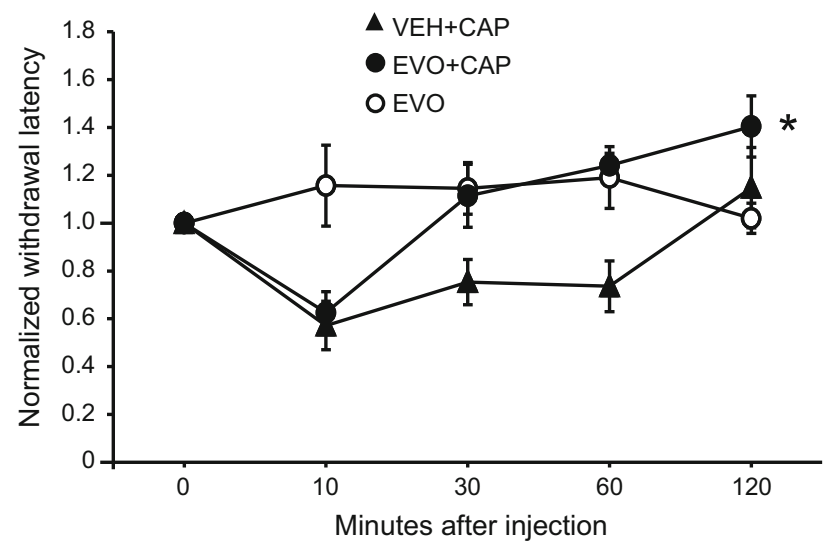

Fig. 6 Evodiamine suppresses capsaicin-induced thermal hyperalgesia in rats. The graph shows the pain response (withdrawal latency to radiant heat) to the subcutaneous application of capsaicin with or without pretreatment of evodiamine. Animals were pretreated subcutaneously with evodiamine $(100 \mu \mathrm{M}, 50 \mu \mathrm{l})$ or vehicle $(5 \%$ DMSO and $0.5 \%$ Tween-20 in saline) $10 \mathrm{~min}$ before the capsaicin $(220 \mu \mathrm{M}$, $50 \mu \mathrm{l})$ or vehicle $(0.5 \%$ Tween-20 in saline) injection. The withdrawal latencies of rats at each time point were normalized to their baseline values (obtained before the application). $E V O+V E H$ evodiamine $(100 \mu \mathrm{M}, 50 \mu \mathrm{l})$ with vehicle $(0.5 \%$ Tween- 20 in saline $)$ injection, $V E H+C A P$ vehicle $(5 \%$ DMSO and $0.5 \%$ Tween-20 in saline) with capsaicin $(220 \mu \mathrm{M}, 50 \mu \mathrm{l})$ injection, $E V O+C A P$ evodiamine $(100 \mu \mathrm{M}, 50 \mu \mathrm{l})$ with capsaicin $(220 \mu \mathrm{M}, 50 \mu \mathrm{l})$ injection. CAP capsaicin, EVO evodiamine. Asterisk $p<0.05, E V O+$ $C A P$ versus $V E H+C A P$, two-way repeated ANOVA followed by Fisher's PLSD. Six rats were used in the $E V O+V E H$ group; seven rats were used in $V E H+C A P$ or $E V O+C A P$ group

\section{Conclusion}

In this study, we demonstrated that evodiamine strongly desensitized TRPV1 channels resulting in the suppression of the capsaicin-induced thermal hyperalgesia response after a tonic pre-application with evodiamine. To our knowledge, these results identify for the first time that the mechanism underlying the analgesic effect of EF or evodiamine may be the activation and subsequent desensitization of TRPV1 in sensory neurons.

Acknowledgments This study was supported by JSPS KAKENHI (23590730), the Heiwa Nakajima Foundation, and the Japan China Medical Association.

Conflict of interest The authors declare that they have no conflict of interest.

\section{References}

1. Caterina MJ, Schumacher MA, Tominaga M, Rosen TA, Levine JD, Julius D (1997) The capsaicin receptor: a heat-activated ion channel in the pain pathway. Nature 389:816-824

2. Hellwig N, Plant TD, Janson W, Schafer M, Schultz G, Schaefer M (2004) TRPV1 acts as proton channel to induce acidification in nociceptive neurons. J Biol Chem 279:34553-34561

3. Brederson JD, Chu KL, Reilly RM, Brown BS, Kym PR, Jarvis MF, McGaraughty S (2012) TRPV1 antagonist, A-889425, inhibits mechanotransmission in a subclass of rat primary afferent neurons following peripheral inflammation. Synapse 66:187-195

4. Amadesi S, Nie J, Vergnolle N, Cottrell GS, Grady EF, Trevisani M, Manni C, Geppetti P, McRoberts JA, Ennes H, Davis JB, Mayer EA, Bunnett NW (2004) Protease-activated receptor 2 sensitizes the capsaicin receptor transient receptor potential vanilloid receptor 1 to induce hyperalgesia. J Neurosci 24:4300-4312

5. Chuang HH, Prescott ED, Kong H, Shields S, Jordt SE, Basbaum AI, Chao MV, Julius D (2001) Bradykinin and nerve growth factor release the capsaicin receptor from PtdIns $(4,5)$ P2-mediated inhibition. Nature 411:957-962

6. Dai Y, Moriyama T, Higashi T, Togashi K, Kobayashi K, Yamanaka H, Tominaga M, Noguchi K (2004) Proteinase-activated receptor 2-mediated potentiation of transient receptor potential vanilloid subfamily 1 activity reveals a mechanism for proteinase-induced inflammatory pain. J Neurosci 24:4293-4299

7. Jin X, Gereau RW (2006) Acute p38-mediated modulation of tetrodotoxin-resistant sodium channels in mouse sensory neurons by tumor necrosis factor-alpha. J Neurosci 26:246-255

8. Khan AA, Diogenes A, Jeske NA, Henry MA, Akopian A, Hargreaves KM (2008) Tumor necrosis factor alpha enhances the sensitivity of rat trigeminal neurons to capsaicin. Neuroscience 155:503-509

9. Caterina MJ, Leffler A, Malmberg AB, Martin WJ, Trafton J, Petersen-Zeitz KR, Koltzenburg M, Basbaum AI, Julius D (2000) Impaired nociception and pain sensation in mice lacking the capsaicin receptor. Science 288:306-313

10. Liao JF, Chiou WF, Shen YC, Wang GJ, Chen CF (2011) Antiinflammatory and anti-infectious effects of Evodia rutaecarpa (Wuzhuyu) and its major bioactive components. Chin Med 6:6

11. Bak EJ, Park HG, Kim JM, Kim JM, Yoo YJ, Cha JH (2010) Inhibitory effect of evodiamine alone and in combination with 
rosiglitazone on in vitro adipocyte differentiation and in vivo obesity related to diabetes. Int $\mathrm{J}$ Obes (Lond) 34:250-260

12. Fei XF, Wang BX, Li TJ, Tashiro S, Minami M, Xing DJ, Ikejima T (2003) Evodiamine, a constituent of Evodiae fructus, induces anti-proliferating effects in tumor cells. Cancer Sci 94:92-98

13. Heo SK, Yun HJ, Yi HS, Noh EK, Park SD (2009) Evodiamine and rutaecarpine inhibit migration by LIGHT via suppression of NADPH oxidase activation. J Cell Biochem 107:123-133

14. Wu CL, Hung CR, Chang FY, Lin LC, Pau KY, Wang PS (2002) Effects of evodiamine on gastrointestinal motility in male rats. Eur J Pharmacol 457:169-176

15. Tsai TH, Lee TF, Chen CF, Wang LC (1995) Thermoregulatory effects of alkaloids isolated from Wu-chu-yu in afebrile and febrile rats. Pharmacol Biochem Behav 50:293-298

16. Pearce LV, Petukhov PA, Szabo T, Kedei N, Bizik F, Kozikowski AP, Blumberg PM (2004) Evodiamine functions as an agonist for the vanilloid receptor TRPV1. Org Biomol Chem 2:2281-2286

17. Wang Z, Sun L, Yu H, Zhang Y, Gong W, Jin H, Zhang L, Liang $\mathrm{H}$ (2012) Binding mode prediction of evodiamine within vanilloid receptor TRPV1. Int J Mol Sci 13:8958-8969
18. Kobayashi Y (2003) The nociceptive and anti-nociceptive effects of evodiamine from fruits of Evodia rutaecarpa in mice. Planta Med 69:425-428

19. Brederson JD, Kym PR, Szallasi A (2013) Targeting TRP channels for pain relief. Eur J Pharmacol 716:61-76

20. Knotkova H, Pappagallo M, Szallasi A (2008) Capsaicin (TRPV1 Agonist) therapy for pain relief: farewell or revival? Clin J Pain $24: 142-154$

21. Moran MM, McAlexander MA, Biro T, Szallasi A (2011) Transient receptor potential channels as therapeutic targets. Nat Rev Drug Discov 10:601-620

22. Dai Y, Iwata K, Fukuoka T, Kondo E, Tokunaga A, Yamanaka H, Tachibana T, Liu Y, Noguchi K (2002) Phosphorylation of extracellular signal-regulated kinase in primary afferent neurons by noxious stimuli and its involvement in peripheral sensitization. J Neurosci 22:7737-7745 